\title{
Conductance fingerprints of noncollinear magnetic states in single-atom contacts: A first-principles Wannier-functions study
}

\author{
Björn Hardrat, ${ }^{1}$ Frank Freimuth, ${ }^{2}$ Stefan Heinze, ${ }^{1}$ and Yuriy Mokrousov ${ }^{2, *}$ \\ ${ }^{1}$ Institut für Theoretische Physik und Astrophysik, Christian-Albrechts-Universität zu Kiel, Leibnizstrasse 15, D-24098 Kiel, Germany \\ ${ }^{2}$ Peter Grünberg Institut and Institute for Advanced Simulation, Forschungszentrum Jülich and JARA, D-52425 Jülich, Germany
}

(Received 7 August 2012; revised manuscript received 21 September 2012; published 31 October 2012)

\begin{abstract}
We present a first-principles computational scheme for investigating the ballistic transport properties of onedimensional nanostructures with noncollinear magnetic order. The electronic structure is obtained within density functional theory as implemented in the full-potential linearized augmented plane-wave method and mapped to a tight-binding-like transport Hamiltonian via noncollinear Wannier functions. The conductance is then computed based on the Landauer formula using the Green's function method. As a first application, we study the conductance between two ferromagnetic Co monowires terminated by single $\mathrm{Mn}$ apex atoms as a function of Mn-Mn separation. We vary the Mn-Mn separation from the contact (about 2.5 to $5 \AA$ ) to the far tunneling regime (5 to $10 \AA$ ). The magnetization direction of the Co electrodes is chosen either in parallel or antiparallel alignment and we allow for different spin configurations of the two Mn spins. In the tunneling and into the contact regime, the conductance is dominated by $s-d_{z^{2}}$ states. In the close contact regime (below $3.5 \AA$ ), there is an additional contribution for a parallel magnetization alignment from the $d_{x z}$ and $d_{y z}$ states which give rise to an increase of the magnetoresistance as it is absent for antiparallel magnetization. If we allow the Mn spins to relax, a noncollinear spin state is formed close to contact due to the competition of ferromagnetic coupling between $\mathrm{Mn}$ and $\mathrm{Co}$ and antiferromagnetic coupling between the Mn spins. We demonstrate that the transition from a collinear to such a noncollinear spin structure as the two $\mathrm{Mn}$ atoms approach leaves a characteristic dip in the distance-dependent conductance and magnetoresistance of the junction. We explain this modification of the spin-valve effect due to the noncollinear spin state based on the spin-dependent hybridization between the $d_{x z, y z}$ states of the Mn spins and their coupling to the Co electrodes.
\end{abstract}

DOI: 10.1103/PhysRevB.86.165449

PACS number(s): 73.63.-b, 73.23.Ad

\section{INTRODUCTION}

Break-junction experiments have allowed to perform transport studies on nanoscale metallic contacts in which the mean-free path of the electrons is much larger than the junction length. The observation of quantized conductance in such systems is a hallmark of ballistic transport and opened new vistas to study the scaling of electronic devices down to the atomic length scale. ${ }^{1}$ A drawback of such experiments is the limited control of the microscopic arrangement in the junction which hinders a straightforward interpretation of the data and makes a comparison with theoretical calculations difficult. ${ }^{2}$ In this respect, a great advantage is given by the use of scanning tunneling microscopy (STM) experiments, in which a tip can approach and contact single atoms or molecules on a surface. ${ }^{3-8}$ In such experiments, it has been possible to measure the conductance as a function of tip-sample distance from the tunneling to the contact regime. Due to the promise of spintronic devices for future applications with low power consumption and high speed, a recent focus of such contact measurements has been magnetic systems, e.g., spin-valve behavior has been observed in single magnetic molecules or atoms on surfaces ${ }^{8,9}$ and the occurrence of the Kondo effect has been found in ferromagnetic atomic contacts. ${ }^{10}$

It has been emphasized that the low coordination of the contact atoms in nanoscale junctions leads to an enhanced tendency towards magnetism, e.g., magnetic moments are formed in systems of otherwise nonmagnetic materials. ${ }^{11-15}$ Naturally, transport phenomena in such magnetic low-dimensional systems have raised a lot of attention and triggered many theoretical studies, which mainly focused on systems with collinear magnetic order, considering also the effect of magnetoresistance. ${ }^{16-21}$ It was recently pointed out that if the magnetization direction of the two electrodes is opposite, a domain wall can form in the contact between them and the noncollinear order in the domain strongly affects the conductance and the magnetoresistance. ${ }^{22-24}$ Finally, the effect of spin-orbit coupling on the conductance needs to be considered, ${ }^{16,25}$ which leads to novel transport phenomena such as the ballistic anisotropic magnetoresistance ${ }^{16,26}$ or the tunneling anisotropic magnetoresistance. ${ }^{27,28}$

Recently, the transition regime from tunneling to contact in a spin-polarized STM geometry has been studied based on density functional theory in order to explain, e.g., the conductance of a single magnetic atom, ${ }^{5}$ and to analyze the contribution from different conduction channels. ${ }^{21}$ As a magnetic STM tip approaches a single magnetic atom on a surface to measure the distance-dependent conductance (as in Ref. 8), an exchange interaction with the tip apex atom occurs. In principle, it is possible to switch the magnetic moment of the adatom in such a way. ${ }^{29}$ If the magnetic moment of the adatom is exchange coupled to the substrate (as in Ref. 8), there is a competition of exchange interactions which can result in a canting of the spins close to contact. Noncollinear spin alignment in such an atomic contact can also occur if the adatom spin is canted due to exchange coupling on a substrate with a spin spiral structure as in Refs. 30 and 31. The noncollinear spin alignment between tip apex atom and adatom can influence the conductance measured with an STM. It may be the origin of the nontrivial 
behavior of the distance-dependent magnetoresistance found experimentally by STM far in the contact regime (Ref. 8). Here, we use first-principles calculations to elucidate the effect of noncollinear spin states on the conductance and on the spin-valve effect of single-atom contacts.

We introduce an approach to calculate the conductance in magnetic nanojunctions with noncollinear spin structure from first principles, employing the methodology of noncollinear Wannier functions (WFs), which we describe in detail. In order to start from an accurate description of the electronic and magnetic structures of the system, we use the full-potential linearized augmented plane-wave (FLAPW) method based on density functional theory. We map the electronic structure of a system in a noncollinear magnetic state from the FLAPW description to a tight-binding-like Hamiltonian via WFs. Finally, we calculate the conductance within the Landauer approach with the technique of Green's functions.

As a model system, we consider two Co monowires to each of which a single apex $\mathrm{Mn}$ atom is attached. We vary the distance between the two $\mathrm{Mn}$ atoms in order to calculate the conductance from the tunneling to the contact regime. The magnetization direction of the two Co electrodes is chosen either parallel (P) or antiparallel (AP), which allows us to obtain the distance-dependent magnetoresistance. In the tunneling regime, the conductance is dominated by states of $s-d_{z^{2}}$-orbital character and only in the contact regime there is an additional contribution due to $d_{x z, y z}$ states. As the latter conduction channel is suppressed in the AP alignment, the magnetoresistance displays a large rise close to contact.

When the two Mn atoms approach in the P electrode alignment, a competition of the exchange interactions between the two Mn spins and between the Mn spins and the Co electrodes occurs. While the Mn spins couple ferromagnetically to the Co electrodes, they couple antiferromagnetically with each other. As a result, a noncollinear arrangement becomes the magnetic ground state and the angle between the two Mn spins changes gradually from $0^{\circ}$ to about $105^{\circ}$ at the closest separation we considered. The conductance displays a characteristic dip as the noncollinear state forms, which is also apparent in the distance-dependent magnetoresistance. We explain this reduction of the conductance due to noncollinear spin states from the spin-dependent hybridization of $d_{x z, y z}$ states between the two Mn atoms which depends on the angle between their spin moments and partly suppresses the conduction in this channel.

The paper is organized as follows. In Sec. II, we introduce our method to calculate the conductance of a one-dimensional nanoscale junction with a noncollinear spin structure. We discuss the extension of Wannier functions to systems with noncollinear order (Sec. II A), the implementation within the FLAPW method (Sec. II B), and the incorporation into our transport code (Sec. II C). In Sec. III, we introduce our model system consisting of two Co monowires to each of which a single $\mathrm{Mn}$ atom is attached. First, we analyze the magnetic and transport properties of collinear spin states from tunneling to contact (Sec. III B) before we address the occurrence of noncollinear spin states in the contact regime (Sec. III C). We analyze the ballistic conductance of such spin states (Sec. III D) and show that a characteristic fingerprint is observed in the distance-dependent conductance and the magnetoresistance (Sec. III E). We end with a summary in Sec. IV.

\section{METHOD}

The density functional theory ${ }^{32}$ (DFT) states that the energy functional of a general magnetic system $E[n(\mathbf{r}), \mathbf{m}(\mathbf{r})]$ is uniquely determined by the charge density $n(\mathbf{r})$ and the magnetization density $\mathbf{m}(\mathbf{r})$. The most common approximation made to a general magnetic system is to assume a collinear magnetization density, i.e., $\mathbf{m}(\mathbf{r})=m(\mathbf{r}) \hat{\mathbf{e}}$, where $\hat{\mathbf{e}}$ is an arbitrary direction. Within this collinear approximation, the energy is a unique functional of the charge density $n(\mathbf{r})$ and the scalar magnetization density $m(\mathbf{r})$. Due to decoupled spin and real space, the spin channels can be treated independently. However, it is known that relaxing the collinear approximation and allowing for noncollinearity of the magnetization density in real space in the DFT setup leads to an ability of reliably treating whole classes of new phenomena, which rely on the properties of complex magnetic states. ${ }^{33}$

\section{A. Noncollinear Wannier functions}

Within the DFT formulation for noncollinear magnetic systems, one solves the Kohn-Sham equations ${ }^{33}$

$$
\left\{-\frac{\hbar^{2}}{2 m_{e}} \nabla^{2} \mathbf{I}_{2}+\mathbf{V}\right\}\left|\boldsymbol{\psi}_{\mathbf{k} m}\right\rangle=\boldsymbol{\epsilon}_{m}(\mathbf{k})\left|\boldsymbol{\psi}_{\mathbf{k} m}\right\rangle,
$$

where $\mathbf{I}_{2}$ is the $2 \times 2$ unity matrix, $\mathbf{V}$ is the potential matrix which also mixes the spin channels, $m$ is the band index, and $\left|\psi_{\mathbf{k} m}\right\rangle=\left(\left|\psi_{\mathbf{k} m \uparrow}\right\rangle,\left|\psi_{\mathbf{k} m \downarrow}\right\rangle\right)^{T}$ is the spinor Bloch function with spin-up and -down components $\left|\psi_{\mathbf{k} m \uparrow}\right\rangle$ and $\left|\psi_{\mathbf{k} m \downarrow}\right\rangle$, respectively.

For the $M$ converged spinor Kohn-Sham orbitals $\left|\boldsymbol{\psi}_{\mathbf{k} m}\right\rangle$ on a uniform mesh of $\mathcal{N}$ k points, the orthonormal set of Wannier functions can be obtained via the transformation ${ }^{34}$

$$
\left|\mathbf{W}_{\mathbf{R} n}\right\rangle=\frac{1}{\mathcal{N}} \sum_{\mathbf{k}} e^{-i \mathbf{k} \cdot \mathbf{R}} \sum_{m=1}^{M} U_{m n}^{(\mathbf{k})}\left|\boldsymbol{\psi}_{\mathbf{k} m}\right\rangle,
$$

where the number of WFs $N$ is smaller than or equal to $M$ and the matrices $U_{m n}^{(\mathbf{k})}$ represent the gauge freedom of the WFs. In the case when $N=M$ and the group of bands we are extracting the WFs from is isolated from other bands, the $U_{m n}^{(\mathbf{k})}$ matrices are unitary at each $\mathbf{k}$ point. Imposing the constraint of maximal localization of WFs in real space determines the set of $U_{m n}^{(\mathbf{k})}$ matrices up to a common global phase, and the corresponding WFs are called maximally localized Wannier functions (MLWFs). ${ }^{35}$ Like the Kohn-Sham orbitals, the MLWFs are spinors and can be written as $\left|\boldsymbol{W}_{\mathbf{k} m}\right\rangle=\left(\left|W_{\mathbf{k} m \uparrow}\right\rangle,\left|W_{\mathbf{k} m \downarrow}\right\rangle\right)^{T}$ in terms of their spin-up and -down components $\left|W_{\mathbf{k} m \uparrow}\right\rangle$ and $\left|W_{\mathbf{k} m \downarrow}\right\rangle$, respectively. Such spinor-valued WFs were introduced and analyzed in detail by Freimuth et al. in Ref. 36 for the case of a system with spin-orbit coupling.

For the construction of MLWFs within DFT electronic-structure codes, the matrix elements $M_{m n}^{(\mathbf{k}, \mathbf{b})}=\left\langle\psi_{\mathbf{k} m}\left|e^{-i \mathbf{b} \cdot \hat{\mathbf{r}}}\right| \psi_{\mathbf{k}+\mathbf{b} n}\right\rangle \quad$ and $\quad A_{m n}^{(\mathbf{k})}=\left\langle\psi_{\mathbf{k} m} \mid g_{n}\right\rangle$ need to be computed, where $\left|g_{n}\right\rangle$ is a localized orbital, which defines the starting point of the iterative procedure of determining the MLWFs. ${ }^{35}$ Since spin up and down are coupled in noncollinear calculations, these matrix elements involve a summation over the spin $\sigma$. 
The matrix elements $\mathbf{H}_{n n^{\prime}}\left(\mathbf{R}_{1}-\mathbf{R}_{2}\right)$ for the WFs tightbinding Hamiltonian

$$
\hat{\mathbf{H}}_{\mathrm{WFs}}=\sum_{\mathbf{R}_{1} n} \sum_{\mathbf{R}_{2} n^{\prime}} \mathbf{H}_{n n^{\prime}}\left(\mathbf{R}_{1}-\mathbf{R}_{2}\right)\left|\mathbf{W}_{\mathbf{R}_{1} n}\right\rangle\left\langle\mathbf{W}_{\mathbf{R}_{2} n^{\prime}}\right|
$$

are given by ${ }^{36}$

$$
\begin{aligned}
\mathbf{H}_{n n^{\prime}}\left(\mathbf{R}_{1}-\mathbf{R}_{2}\right) & =\frac{1}{\mathcal{N}} \sum_{\mathbf{k} m} \boldsymbol{\epsilon}_{m}(\mathbf{k})\left\langle\mathbf{W}_{\mathbf{R}_{1} n} \mid \boldsymbol{\psi}_{\mathbf{k} m}\right\rangle\left\langle\boldsymbol{\psi}_{\mathbf{k} m} \mid \mathbf{W}_{\mathbf{R}_{2} n^{\prime}}\right\rangle \\
& =\frac{1}{\mathcal{N}} \sum_{\mathbf{k} m} \boldsymbol{\epsilon}_{m}(\mathbf{k}) e^{i \mathbf{k} \cdot\left(\mathbf{R}_{1}-\mathbf{R}_{2}\right)}\left(U_{m n}^{(\mathbf{k})}\right)^{*}\left(U_{m n^{\prime}}^{(\mathbf{k})}\right) .
\end{aligned}
$$

Even though the Wannier and Bloch functions are spinor valued, the transformation of the Hamiltonian from Bloch into Wannier representation is fully determined by the matrices $U_{m n}^{(\mathbf{k})}$ and the eigenvalues $\boldsymbol{\epsilon}_{m}(\mathbf{k})$ as in the collinear case.

\section{B. Noncollinear Wannier functions within the FLAPW method}

The treatment of noncollinear magnetism within the FLAPW method as implemented in the Jülich DFT code FLEUR (Refs. 33 and 37) neglects the effect of intra-atomic noncollinearity. Space is partitioned into the muffin-tin (MT) and interstitial regions (IR). The spin density $\mathbf{m}(\mathrm{r})$ in the IR is treated without shape approximation as a continuous vector field. In the MT sphere $\mathrm{MT}^{\alpha}$ of atom $\alpha$ only the projection of the spin density onto the direction $\hat{\mathbf{e}}_{M}^{\alpha}$ of the average spin moment is used for the generation of the exchangecorrelation potential. The explicit one- and two-dimensional implementations also contain a third region, the vacuum region (VR), which can be treated analogously to the IR. ${ }^{38,39}$ Thus, the self-consistent spin density is approximated as

$$
\mathbf{m}(\mathbf{r})= \begin{cases}\mathbf{m}(\mathbf{r}) & \mathrm{IR}(\mathrm{VR}), \\ m^{\alpha}(\mathbf{r}) \hat{\mathbf{e}}_{M}^{\alpha} & \mathrm{MT}^{\alpha} .\end{cases}
$$

A part of the intra-atomic noncollinearity can still be described within this hybrid approach by decreasing the MT radii.

The radial solutions $u_{l \sigma^{\alpha}}^{\alpha}(r)$ of angular momentum $l$ of the scalar-relativistic Schrödinger equation in $\mathrm{MT}^{\alpha}$ and their energy derivatives $\dot{u}_{l \sigma^{\alpha}}^{\alpha}(r)$ are calculated for the two spins $\sigma^{\alpha}$ and used for the expansion of basis functions and Bloch functions. The spin quantum number $\sigma^{\alpha}$ refers to the local spin quantization axis $\hat{\mathbf{e}}_{M}^{\alpha}$. The expansion coefficients of the eigenspinors of the local-spin-quantization axis in terms of the eigenspinors of the global-spin-quantization axis, which is the $z$ axis, are given by

$$
\begin{aligned}
& \chi_{\uparrow}^{\alpha g}=\left[\exp \left(-i \frac{\phi}{2}\right) \cos \left(\frac{\theta}{2}\right), \exp \left(i \frac{\phi}{2}\right) \sin \left(\frac{\theta}{2}\right)\right]^{T}, \\
& \chi_{\downarrow}^{\alpha g}=\left[-\exp \left(-i \frac{\phi}{2}\right) \sin \left(\frac{\theta}{2}\right), \exp \left(i \frac{\phi}{2}\right) \cos \left(\frac{\theta}{2}\right)\right]^{T},
\end{aligned}
$$

where $\phi$ and $\theta$ are azimuthal and polar angles of the spin direction of $\mathrm{MT}^{\alpha}$ with respect to the global frame $g$.

Within the MTs, the wave function $\psi_{\mathbf{k} m}(\mathbf{r})$ is thus given by

$$
\begin{aligned}
\left.\psi_{\mathbf{k} m}(\mathbf{r})\right|_{\mathrm{MT}^{\alpha}}= & \sum_{\sigma^{\alpha} L}\left[A_{m L \sigma^{\alpha}}^{\alpha}(\mathbf{k}) u_{l \sigma^{\alpha}}^{\alpha}(r)\right. \\
& \left.+B_{m L \sigma^{\alpha}}^{\alpha}(\mathbf{k}) \dot{u}_{l \sigma^{\alpha}}^{\alpha}(r)\right] Y_{L}(\hat{\mathbf{r}}) \chi_{\sigma^{\alpha}}^{\alpha g},
\end{aligned}
$$

where $L$ denotes the angular momentum quantum numbers, $Y_{L}$ is the corresponding spherical harmonic, and $m$ is the band index.

Using functions $\left|g_{n}\right\rangle$, which are restricted each to a single MT sphere, has been found to result in a very good starting point for the iterative optimization of collinear WFs. ${ }^{36}$ Due to the approximate intra-atomic collinearity, it is reasonable to choose in the noncollinear case the localized orbitals $\left|g_{n}\right\rangle$ to be eigenstates of the projection of the spin operator onto the local-spin-quantization axis $\hat{\mathbf{e}}_{M}^{\alpha}$ :

$$
\left.g_{n}(\mathbf{r})\right|_{\mathrm{MT}^{\alpha(n)}}=\sum_{L} c_{n L} \tilde{u}_{n l}(r) Y_{L}(\hat{\mathbf{r}}) \chi_{\sigma(n)}^{\alpha(n) g} .
$$

Here, $c_{n L}$ are expansion coefficients, $\alpha(n)$ is the index of the atom for which $g_{n}(r)$ is nonzero, $\sigma(n)$ is the spin associated with this trial orbital $g_{n}(\mathbf{r})$, and $\tilde{u}_{n l}(r)$ is the radial part of the trial orbital. Thus, we obtain

$$
\begin{aligned}
A_{m n}^{(\mathbf{k})}= & \sum_{L} c_{n L}\left\{\left[A_{m L \sigma(n)}^{\alpha}(\mathbf{k})\right]^{*} \int u_{l \sigma(n)}^{\alpha(n)}(r) \tilde{u}_{n l}(r) r^{2} d r\right. \\
& \left.+\left[B_{m L \sigma(n)}^{\alpha}(\mathbf{k})\right]^{*} \int \dot{u}_{l \sigma(n)}^{\alpha(n)}(r) \tilde{u}_{n l}(r) r^{2} d r\right\} .
\end{aligned}
$$

The MT contribution to the $M_{m n}^{(\mathbf{k}, \mathbf{b})}$ matrix may be written as

$$
\left.M_{m n}^{(\mathbf{k}, \mathbf{b})}\right|_{\mathrm{MT}}=\sum_{\alpha, \sigma^{\alpha}} \int_{\mathrm{MT}^{\alpha}} d^{3} r\left[\psi_{\mathbf{k} m \sigma^{\alpha}}(\mathbf{r})\right]^{*} e^{-i \mathbf{b} \cdot \mathbf{r}} \psi_{\mathbf{k}+\mathbf{b} n \sigma^{\alpha}}(\mathbf{r}),
$$

with $\psi_{\mathbf{k} m \sigma^{\alpha}}(\mathbf{r})$ given by

$$
\psi_{\mathbf{k} m \sigma^{\alpha}}(\mathbf{r})=\sum_{L} Y_{L}(\hat{\mathbf{r}})\left[A_{m L \sigma^{\alpha}}^{\alpha}(\mathbf{k}) u_{l \sigma^{\alpha}}^{\alpha}(r)+B_{m L \sigma^{\alpha}}^{\alpha}(\mathbf{k}) \dot{u}_{l \sigma^{\alpha}}^{\alpha}(r)\right] .
$$

The computation of the $M_{m n}^{(\mathbf{k}, \mathbf{b})}$ matrix for noncollinear systems reduces therefore to integrals for which explicit expressions have been given for the FLAPW method. ${ }^{36}$

\section{Ballistic transport in systems with noncollinear magnetism}

The extension of the collinear scheme for ballistic transport, which we described in detail in our previous publication (Ref. 16) to a noncollinear setup is now rather straightforward. Given the minimal WFs Hamiltonian $\hat{\mathbf{H}}_{\mathrm{WFs}}$ [Eq. (3)] for a noncollinear system, we are able to construct the tight-binding Hamiltonian of the nanojunction in accordance to our transport method, ${ }^{16}$ which employs the partitioning of space into the scattering region $(S)$, as well as left $(L)$ and right $(R)$ leads:

$$
\mathbf{H}=\left(\begin{array}{ccc}
\mathbf{H}_{L} & \mathbf{H}_{L S} & \mathbf{0} \\
\mathbf{H}_{L S}^{\dagger} & \mathbf{H}_{S} & \mathbf{H}_{R S}^{\dagger} \\
\mathbf{0} & \mathbf{H}_{R S} & \mathbf{H}_{R}
\end{array}\right) .
$$

Compared to a collinear calculation, we have to deal with twice as many Wannier functions due to the inseparable spin channels. Two calculations using the locking technique ${ }^{16}$ are required for Eq. (12). The Hamiltonian matrix $\mathbf{H}_{S}$ and the matrices $\mathbf{H}_{L S}$, describing the coupling to the leads, are obtained from a supercell FLAPW calculation. The Green's functions for the 
leads $\mathbf{G}_{L / R}(E)=\left[(E+i \epsilon) \mathbf{I}_{L / R}-\mathbf{H}_{L / R}\right]^{-1}$ can be brought to finite-sized surface Green's functions $\mathbf{g}_{L / R}$ by constructing $\mathbf{H}_{L / R}$ based on principal layers $\mathbf{h}_{L / R}$ and the coupling matrices $\mathbf{h}_{L L / R R} .{ }^{16}$ Those matrices are obtained from a separate calculation of a perfect periodic lead. Following our LandauerBüttiker method, the noncollinear ballistic transport can be calculated with the Green's function of the scattering region:

$$
\mathbf{G}_{S}(E)=\left[E \mathbf{I}_{S}-\mathbf{H}_{S}-\mathbf{H}_{L S}^{\dagger} \mathbf{g}_{L} \mathbf{H}_{L S}-\mathbf{H}_{R S}^{\dagger} \mathbf{g}_{R} \mathbf{H}_{R S}\right]^{-1} \text {. }
$$

The interaction between scattering region and the leads, and the resulting level broadenings, are described by broadening matrices $\Gamma$ :

$$
\Gamma_{L / R}(E)=i\left[\Sigma_{L / R}(E)-\Sigma_{L / R}^{\dagger}(E)\right],
$$

where $\Sigma_{L / R}(E)$ are the self-energies of the leads:

$$
\Sigma_{L / R}(E)=\mathbf{H}_{L S / R S}^{\dagger} \mathbf{g}_{L / R}(E) \mathbf{H}_{L S / R S} .
$$

Finally, the ballistic transport process is described by the transmission function $T(E)$,

$$
T(E)=\operatorname{Tr}\left[\mathbf{G}_{S}(E) \boldsymbol{\Gamma}_{L}(E) \mathbf{G}_{S}^{\dagger}(E) \boldsymbol{\Gamma}_{R}(E)\right],
$$

resulting in the conductance through the junction

$$
G(E)=\frac{e^{2}}{h} T(E)=\frac{1}{2} G_{0} T(E)
$$

with the conductance quantum $G_{0}=2 e^{2} / h$. In the noncollinear case, the trace operation of Eq. (16) has to be additionally performed over the spin $\sigma$. The spin-channel information is therefore lost for general noncollinear systems.

We tested noncollinear Wannier functions on freestanding noncollinear magnetic $\mathrm{Mn}$ chains and found them to reproduce the FLAPW electronic structure with any given accuracy. For the performed transport calculations, a second-nearestneighbor (NN) tight-binding-like Hamiltonian is sufficient due to the excellent correspondence of FLAPW and WFs electronic structure in the vicinity of the Fermi level in absence of $s-d_{z^{2}}$ band edges in that particular region, which usually would require to consider more neighbors. ${ }^{16}$ For the system, considered in the following, the orbitals participating in transport can be arranged according to the symmetry into the $\Delta_{1}$ ( $s$ and $d_{z^{2}}$ orbitals), the $\Delta_{3}\left(d_{x z / y z}\right)$, and the $\Delta_{4}\left(d_{x y / x^{2}-y^{2}}\right)$ groups.

\section{THE $\mathrm{Co}_{\infty}-\mathrm{Mn}-\mathrm{Mn}-\mathrm{Co}_{\infty}$ JUNCTION}

In the following section, we investigate the ballistic transport properties of collinear and noncollinear magnetic configurations of a $\mathrm{Co}_{\infty}-\mathrm{Mn}-\mathrm{Mn}-\mathrm{Co}_{\infty}$ junction, consisting of semi-infinite ferromagnetic Co monowires with magnetic Mn "tip" atoms (see sketch of the structure in Fig. 1). We will discuss the effect of noncollinear magnetism on ballistic transport through such a junction, specifically keeping in mind tunneling-to-contact STM (Refs. 3-8) and mechanically controllable break-junctions ${ }^{40}$ experiments. In particular, we investigate the changes in the transport properties upon changing the distance between the two Mn atoms, while keeping all other interatomic distances fixed at their equilibrium "semi-infinite" values. We will show that upon bringing the leads together, the noncollinearity in this system emerges as a result of competing $\mathrm{Mn}-\mathrm{Mn}$ and $\mathrm{Mn}-\mathrm{Co}$ exchange interactions.

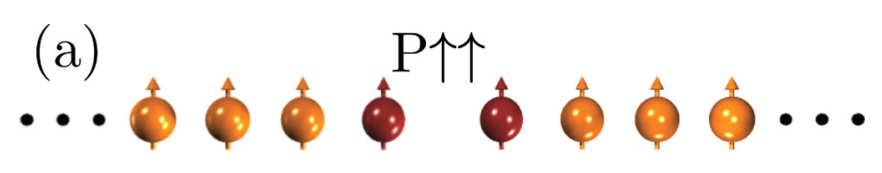

$\cdots \oplus \oplus \oplus_{\alpha-\alpha}^{\mathrm{P} \alpha} \oplus \phi \cdots$ $\cdots \oplus \phi \phi^{\mathrm{P} H} \phi \phi \phi \cdots$ $\cdots \phi \phi \phi^{\mathrm{P} \downarrow \uparrow} \phi \phi \phi \cdots$

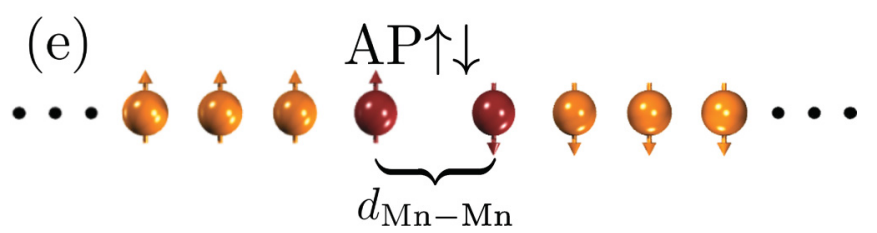

FIG. 1. (Color online) Calculated magnetic configurations in the $\mathrm{Co}_{\infty}-\mathrm{Mn}-\mathrm{Mn}-\mathrm{Co}_{\infty}$ junction. The magnetization of the left lead always points up $(\uparrow)$. The magnetization of the right lead can be either parallel (P) or antiparallel (AP) to it. The direction of the Mn spins is marked with respect to the left lead: pointing up ( $\uparrow)$, down $(\downarrow)$, or along a direction at an angle $\alpha$ with the magnetization of the left lead. Special configurations of interest which are discussed in the text are shown: (a) $\mathrm{P} \uparrow \uparrow$, (b) symmetric $\mathrm{P} \alpha$ state with $\alpha=105^{\circ}$, $\mathrm{P} 105^{\circ}$, (c) $\mathrm{P} \downarrow \downarrow$, (d) $\mathrm{P} \downarrow \uparrow$, and (e) AP $\uparrow \downarrow$. In the text, we refer to the separation between the leads in terms of the distance between the Mn atoms $d_{\mathrm{Mn}-\mathrm{Mn}}$. Co atoms are displayed as orange spheres, Mn atoms are displayed as red spheres.

We then explore the influence of noncollinear magnetism on ballistic transport for various collinear and noncollinear configurations. The nomenclature for the magnetic states in the $\mathrm{Co}_{\infty}-\mathrm{Mn}-\mathrm{Mn}-\mathrm{Co}_{\infty}$ junction includes the alignment of the magnetization directions of the leads, parallel $(\mathrm{P})$ or antiparallel (AP) to each other, and the directions of the two Mn spins. Without loss of generality, these directions are denoted with respect to the left lead, which has magnetization "up." The Mn spins can point "up" ( $\uparrow)$, "down" $(\downarrow)$, or in a direction which makes an angle $\alpha$ with the direction up (see Fig. 1). In the latter case, we consider the symmetric configuration, denoted as $\mathrm{P} \alpha$, in which the spins of the $\mathrm{Mn}$ atoms make an angle of $2 \alpha$ between each other. For all considered noncollinear $\mathrm{P} \alpha$ states, we fixed the direction of all Co atoms either up or down, depending on the magnetization direction of the corresponding lead. The energy differences between different magnetic states are given per Mn atom.

\section{A. Computational details}

For all collinear and noncollinear electronic-structure calculations, we used density functional theory within generalized gradient approximation (GGA) to the exchange-correlation potential, ${ }^{41}$ as implemented in the FLAPW Jülich code 
FLEUR. ${ }^{37}$ The wires were calculated in three-dimensional supercells, with an interchain separation in the $x-y$ plane of 13 bohr. The supercell setup along the chain's axes ( $z$ direction) is described in detail below. The Brillouin zone (BZ) was sampled by 12 or $24 \mathbf{k}$ points along the $z$ axis, depending on the size of the supercell. All calculations were performed with an LAPW basis cutoff parameter $k_{\max }$ of $3.7 \mathrm{bohr}^{-1}$, resulting in approximately $625 \mathrm{LAPW}$ basis functions per atom.

The parallel magnetic configuration (P) of $\mathrm{Co}_{\infty}-\mathrm{Mn}-\mathrm{Mn}-$ $\mathrm{Co}_{\infty}$ junctions was investigated in an eight-atom supercell along the chain direction, consisting of six Co atoms with an equilibrium interatomic distance of the Co infinite monowire of $d_{\mathrm{Co}}=4.15$ bohr, and two attached Mn atoms (see Fig. 1). For all considered magnetic configurations, with parallel or antiparallel alignment of the magnetization of the leads, as well as noncollinear magnetic states, irrespective of the separation between the leads, we fixed the Co-Mn distance $d_{\mathrm{Co}-\mathrm{Mn}}$ to $4.48 \mathrm{bohr}$, which corresponds to the equilibrium distance between the ferromagnetic Co and $\mathrm{Mn}$ atoms at a very large separation between the leads. For the $\mathrm{P} \uparrow \uparrow$ and $\mathrm{P} \downarrow \downarrow$ states of the junction, we considered the inter-Mn separation of $d_{\mathrm{Mn}-\mathrm{Mn}}=5.0,5.5,7.0,8.5,10.0,12.5,15.0,17.5$, and 20.0 bohr. The antiparallel magnetic configuration (AP) of $\mathrm{Co}_{\infty}-\mathrm{Mn}-\mathrm{Mn}-\mathrm{Co}_{\infty}$ junctions was calculated in a 16-atom supercell, consisting of $6 \uparrow-\mathrm{Co}$ atoms and $2 \mathrm{Mn}$ atoms on one side, and $6 \downarrow$-Co atoms and $2 \mathrm{Mn}$ atoms at another end of the junction. In this case, $d_{\mathrm{Mn}-\mathrm{Mn}}$ was set to $4.5,5.0,5.5,7.0$, and 8.5 bohr.

For the conductance calculations we applied the locking technique to a perfect monowire to describe the semi-infinite leads, as described in detail in Ref. 16. In all cases, the Wannier functions were generated on a $1 \times 1 \times 24$ k-point grid in the BZ. For the collinear cases, the WFs were generated from $14 s$ and $53 d$ orbitals per atom for each spin separately, which were constructed from the radial solutions for the FLAPW potential. In noncollinear calculations, the spin channels are mixed, and $24 s$ and $103 d$ orbitals per atom were used to construct the WFs. The energy bands were disentangled using the procedure described in Ref. 42. For all calculations, we used a frozen energy window of $[-12.0,+1.6] \mathrm{eV}$ with respect to the Fermi energy. Respectively, for the collinear calculations, the lowest 58 eigenvalues per $\mathbf{k}$ point were used to obtain 48 WFs for the 8-atom supercell and the lowest 104 eigenvalues per k point for 96 WFs for the 16-atom supercell calculations. With noncollinearity of the magnetization included, the lowest 103 eigenvalues per $\mathbf{k}$ point were used to obtain 96 WFs for the 8-atom unit cell. The spreads of the noncollinear WFs of Mn atoms vary between the spreads of the corresponding WFs for the parallel and antiparallel alignment of the Mn spins. For example, the spread of the Mn $\Delta_{3}$ WFs smoothly changes from 2.5 to $2.0 \mathrm{bohr}^{2}$ as the angle the Mn spin makes with the $x$ axis is changed between $0^{\circ}$ and $180^{\circ}$. For testing purposes, for several noncollinear configurations we compared the electronic structure of the system calculated with FLEUR and with corresponding WFs. We find that the quality of the description of the band structure of the system with the tight-binding Hamiltonian constructed from noncollinear WFs is in a very close correspondence to that obtained previously for the case of Pt and Co chains, and reported in Figs. 3, 8, and

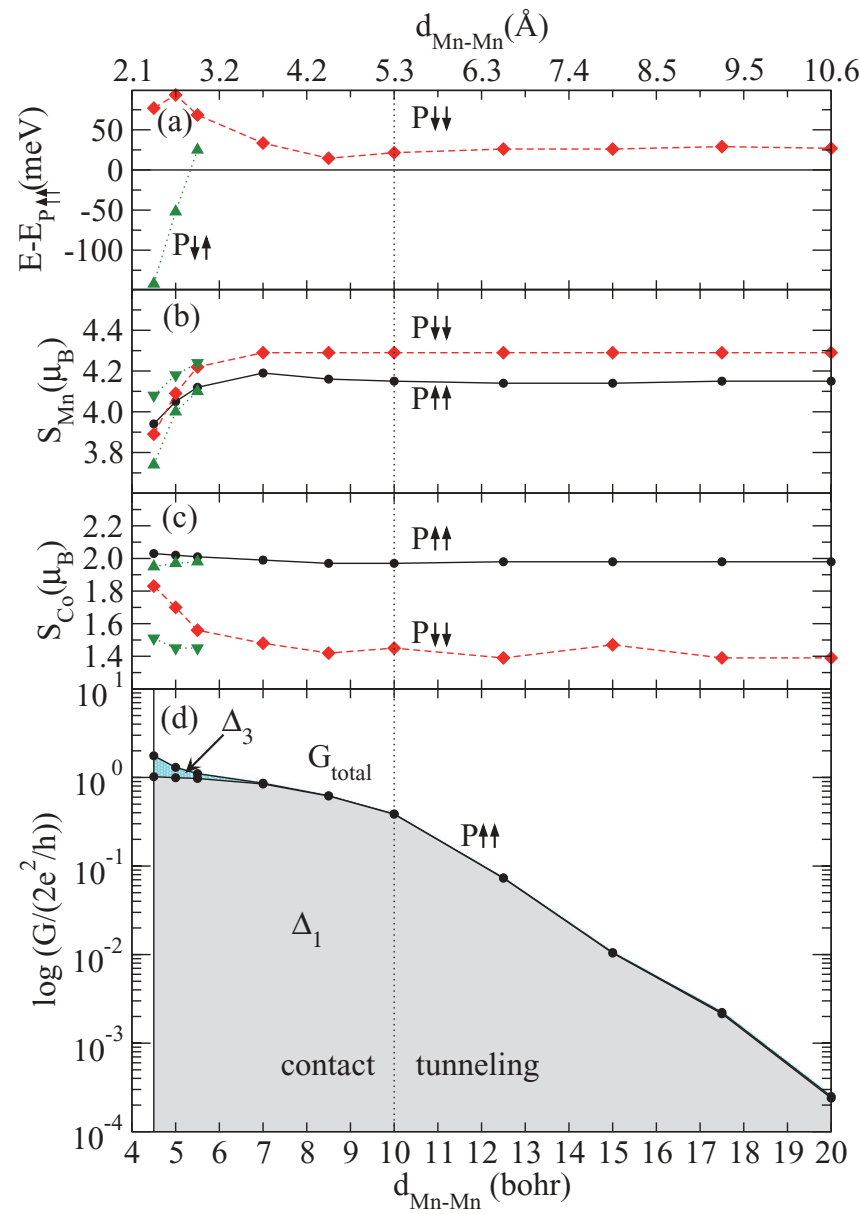

FIG. 2. (Color online) (a) Energy of the $\mathrm{P} \downarrow \downarrow$ state (red diamonds, dashed line) and $\mathrm{P} \downarrow \uparrow$ state (green triangles, dotted line) with respect to the energy of the $\mathrm{P} \uparrow \uparrow$ state, as a function of the Mn-Mn interatomic distance $d_{\mathrm{Mn}-\mathrm{Mn}}$. (b), (c) Spin moment of the Mn atoms $S_{\mathrm{Mn}}(\mathrm{b})$ and $\mathrm{NN}$ Co atoms $S_{\mathrm{Co}}$ (c) as a function of $d_{\mathrm{Mn}-\mathrm{Mn}}$. In (b) and (c), the values are given for the $\mathrm{P} \uparrow \uparrow$ state with black circles (solid line), for the $\mathrm{P} \downarrow \downarrow$ state with red diamonds (dashed line), and for the $\mathrm{P} \downarrow \uparrow$ state with up triangles for the FM side and down triangles for the AFM side of the junction. (d) Total conductance at the Fermi level $G_{\text {total }}\left(E_{F}\right)$ (upper line) and $\Delta_{1}$ conductance $G_{\Delta_{1}}\left(E_{F}\right)$ (lower line) on a logarithmic scale for the $\mathrm{P} \uparrow \uparrow$ state. Gray shaded area is associated with the $\Delta_{1}$ conductance, while cyan shaded area with the $\Delta_{3}$ conductance.

10 of Ref. 16. In particular, we find that a very good description of the electronic structure can be achieved with WFs within the third-nearest-neighbor approximation, while for WFs calculations of the transmission in the vicinity of the Fermi level already the second-nearest-neighbor approximation to the WFs Hamiltonian provides very reliable results.

\section{B. Collinear magnetic states of the junction from tunneling to contact}

We start the investigation of the $\mathrm{Co}_{\infty}-\mathrm{Mn}-\mathrm{Mn}-\mathrm{Co}_{\infty}$ junction with both leads positioned far away from each other. To mimic a tip-sample approach, we decrease the $\mathrm{Mn}-\mathrm{Mn}$ distance $d_{\mathrm{Mn}-\mathrm{Mn}}$, and calculate the energies of the collinear states $\mathrm{P} \uparrow \uparrow$, $\mathrm{P} \downarrow \downarrow$, and $\mathrm{P} \downarrow \uparrow$, showing the results in Fig. 2(a). The energy 


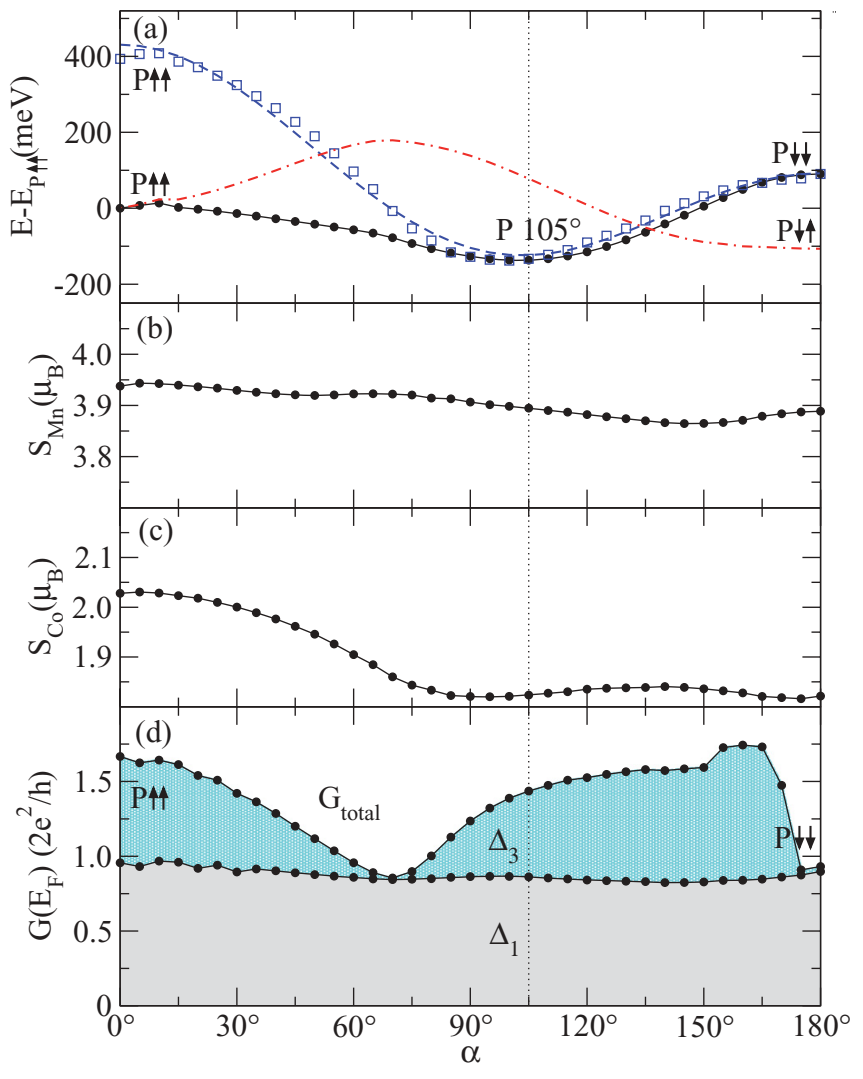

FIG. 3. (Color online) (a) Total energy of the $\mathrm{P} \alpha$ state with respect to the $\mathrm{P} \uparrow \uparrow$ state as a function of angle $\alpha$ (solid line, filled circles). With open black squares, we show the total energy of the $\mathrm{P} \alpha$ state from which the Stoner energy due to creation of the NN Co spin moments is subtracted (relative to the $\mathrm{P} \downarrow \downarrow$ state). The corresponding fit of the corrected energy to the Heisenberg model is shown with a dashed blue line. With the red dotted line, we show the total energy of the P $\uparrow \downarrow$ state with only the $\downarrow$-Mn spin rotated by an angle $\alpha$, calculated within the Heisenberg model and incorporating also the Stoner exchange. (b) Mn spin moment as a function of angle $\alpha$. (c) NN Co spin moment as a function of angle $\alpha$. (d) Ballistic conductance at the Fermi level for the $\mathrm{P} \alpha$ state as a function of angle $\alpha$ : total conductance $G_{\text {total }}\left(E_{F}\right)$ (upper line), the $\Delta_{3}$ conductance contribution (cyan shaded area), and $\Delta_{1}$ conductance $G_{\Delta_{1}}\left(E_{F}\right)$ (lower line, gray shaded area).

difference between $\mathrm{P} \uparrow \uparrow$ and $\mathrm{P} \downarrow \downarrow$ states when the distance is varied in the tunneling regime from $d_{\mathrm{Mn}-\mathrm{Mn}}=20$ bohr down to 10 bohr remains relatively constant and constitutes around $27 \mathrm{meV}$ per atom, indicating weak interaction between both sides of the junction and a weak ferromagnetic coupling between the $\mathrm{Mn}$ atom and its nearest Co neighbor (NN Co). After a small reduction of the energy difference between the $\mathrm{P} \uparrow \uparrow$ and $\mathrm{P} \downarrow \downarrow$ states around $d_{\mathrm{Mn}-\mathrm{Mn}}=8 \mathrm{bohr}$, the ferromagnetic (FM) Mn-Co coupling becomes more stable for decreasing $d_{\mathrm{Mn}-\mathrm{Mn}}$, expressed in an increasing energy difference. In the contact regime, we find a slight decrease in the energy difference from $93.5 \mathrm{meV}$ at $d_{\mathrm{Mn}-\mathrm{Mn}}=5.0 \mathrm{bohr}$ down to $77 \mathrm{meV}$ per atom at $d_{\text {Mn-Mn }}=4.5$ bohr. This decrease in energy can be correlated with strong changes in the Mn and NN Co spin moments, $S_{\mathrm{Mn}}$ and $S_{\mathrm{Mn}}$, respectively, upon decreasing the distance [see Figs. 2(b) and 2(c)] (see also discussion in the next section).
While in all cases the spin moments of the Co atoms, not neighboring the $\mathrm{Mn}$ atoms directly $\left(\approx 2.09 \mu_{B}\right)$, are very similar to the spin moments of the Co atom in an infinite lead $\left(\approx 2.07 \mu_{B}\right)$, the spin moments of $\mathrm{Mn}$ atoms and the NN Co atoms can be strongly affected by $d_{\mathrm{Mn}-\mathrm{Mn}}$ at close contact and the spin configuration of the junction. Namely, for the P $\downarrow \downarrow$ state, $S_{\mathrm{Mn}}$ decreases from 4.3 to $3.9 \mu_{B}$, while NN $S_{\mathrm{Co}}$ increases from 1.4 to $1.8 \mu_{B}$, as $d_{\mathrm{Mn}-\mathrm{Mn}}$ is varied from 5.5 to 4.5 bohr. On the other hand, if the Mn spin moment exhibits a similar variation as a function of distance for the $\mathrm{P} \uparrow \uparrow$ state, the spin moment of the NN Co atoms remains relatively constant $\left(\approx 2 \mu_{B}\right)$. This interplay between structure and magnetism already indicates that the intra-atomic as well as interatomic exchange, given by the Stoner parameter $I$ and the Heisenberg exchange constants $J$, respectively, may be of importance for further understanding of the magnetic properties of this system.

The change from FM coupling at larger interatomic distances to an antiferromagnetic (AFM) coupling at smaller $d_{\mathrm{Mn}-\mathrm{Mn}}$ in an infinite $\mathrm{Mn}$ chain has been previously predicted based on DFT calculations. ${ }^{43}$ In the vicinity of this crossover point, the Mn spins favor noncollinear magnetic order. ${ }^{44-46}$ To demonstrate a strong tendency of $\mathrm{Mn}$ spin moments to AFM coupling at smaller values of $d_{\mathrm{Mn}-\mathrm{Mn}}$, we plot the energy difference between the $\mathrm{P} \uparrow \uparrow$ and $\mathrm{P} \downarrow \uparrow$ states in Fig. 2(a). Reversing one of the Mn spin moments in the $\mathrm{P} \uparrow \uparrow$ configuration is clearly energetically more favorable than the $\mathrm{P} \uparrow \uparrow$ state when the distance between the Mn atoms is below $\approx 5.2$ bohr. In this case, the gain in energy due to switch of the Mn spin moment can be explained only by the strong AFM coupling of the two $\mathrm{Mn}$ atoms for this regime of interatomic distance since the coupling of the Mn atom with its NN Co atom is ferromagnetic. The spin moments in the $\mathrm{P} \downarrow \uparrow$ state at $d_{\mathrm{Mn}-\mathrm{Mn}}=4.5$ bohr constitute $4.1 \mu_{B}$ for $\mathrm{Mn}$ and $1.5 \mu_{B}$ for its NN Co on the AFM side, and $3.7 \mu_{B}$ for Mn and $1.9 \mu_{B}$ for the NN Co on the FM side [see Figs. 2(b) and 2(c)]. For larger $d_{\mathrm{Mn}-\mathrm{Mn}}$ values, the $\mathrm{P} \uparrow \uparrow$ state is the lowest in energy as compared to all possible collinear states of the junction in which the magnetization direction of the left and right leads is the same, which is indicative of the FM Mn-Mn coupling for larger distances.

In Fig. 2(d), we present the results of our calculations for the evolution of the ballistic conductance of a $\mathrm{P} \uparrow \uparrow$ junction when going from the tunneling to the contact regime. The main contribution to the conductance at large $\mathrm{Mn}-\mathrm{Mn}$ distances is coming solely from the $\Delta_{1}$ channel, owing to the overlap between the $s-d_{z^{2}}$ orbitals of the neighboring Mn atoms across the barrier. Within our approach, the expected exponential behavior of the conductance at very large distances is very nicely reproduced. At a distance of $d_{\mathrm{Mn}-\mathrm{Mn}} \approx 10 \mathrm{bohr}$, the conductance approaches the magnitude of the conductance quantum, reaching saturation upon further decreasing the distance. For distances in the contact regime below 7 bohr, more localized $d$ orbitals of $\Delta_{3}$ symmetry start contributing to the total conductance, as can be seen in Fig. 2(d). The $\Delta_{3}$ contribution to the conductance increases with decreasing distance. As we shall see in the following, the details of hybridization between the $\Delta_{3}$ orbitals are very sensitive to the magnetic state of the junction. On the other hand, in all considered cases, the $d$ states of $\Delta_{4}$ symmetry do not contribute 
to the conductance due to an energetic mismatch between the states of this symmetry of NN Co and Mn atoms (see discussion in Sec. III D).

\section{Noncollinear magnetic states of the junction in contact regime}

According to the findings presented above, we expect that Mn spin moments in the contact regime will experience a frustration when the magnetizations of the leads are parallel to each other. In this case, when Mn atoms are close enough, FM coupling of Mn spins with NN Co atoms and AFM Mn-Mn coupling can possibly lead to a stable noncollinear magnetic state. In order to consider this situation, we introduce an angle $\alpha$ between the spin moments of the Mn and the NN Co atoms, rotating the first $\mathrm{Mn}$ spin moment by $+\alpha$ and the second one by $-\alpha$, while keeping the moments of the Co atoms fixed [see Fig. 1(b)]. This is what we call a symmetric $\mathrm{P} \alpha$ state. We choose a distance of 4.5 bohr between the Mn atoms as a representative of the contact regime at which the Mn-Mn coupling is strongly antiferromagnetic.

The results of our calculations for the total energy of the $\mathrm{P} \alpha$ state, $E_{\mathrm{P} \alpha}$, in relation to the energy of the $\mathrm{P} \uparrow \uparrow$ state are shown in Fig. 3(a) as a function of the angle $\alpha$. From this plot we observe that the minimum of the total energy is acquired for the noncollinear $\mathrm{P} 105^{\circ}$ state, which is $137 \mathrm{meV}$ lower in energy than the corresponding collinear $\mathrm{P} \uparrow \uparrow$ state. The failure of a straightforward description of the energy landscape $E_{\mathrm{P} \alpha}$ in terms of a simple Heisenberg model which assumes just the nearest-neighbor Co-Mn and Mn-Mn exchange coupling, given by antiferromagnetic $J_{\mathrm{Mn}}<0$ and ferromagnetic $J_{\mathrm{Co}}>$ 0 , respectively, can be understood from noticing that the expression for the energy within this approximation, given by

$$
E_{\mathrm{P} \alpha}(\alpha)=-\frac{1}{2}\left[J_{\mathrm{Mn}} \cos (2 \alpha)+2 J_{\mathrm{Co}} \cos (\alpha)\right],
$$

acquires a minimum for angles $\alpha$ below $90^{\circ}$, in contradiction to our calculations.

The solution to this deficiency of the Heisenberg model can be given by lifting the assumption that the exchange interaction between the $\mathrm{Mn}$ and Co spins, given by $J_{\mathrm{Co}}$, is ferromagnetic. As we can see from Figs. 3(b) and 3(c), while the Mn spin moment remains relatively constant upon changing $\alpha, S_{\mathrm{Co}}$ for values of $\alpha$ below $60^{\circ}$ is by as much as $0.2 \mu_{B}$ larger than for $\alpha>90^{\circ}$. Owing to the intra-atomic Stoner exchange, the noncollinear states with small $\alpha$ therefore acquire a negative contribution to the total energy in addition to that proportional to $J_{\mathrm{Co}}$, as compared to larger angles. If we account for the energy gain due to creation of the NN Co spin moments by a Stoner parameter of Co, $I \approx 990 \mathrm{meV},{ }^{47}$ and subtract the energy gain $E_{\mathrm{Stoner}}=\frac{1}{2} I S_{\mathrm{Co}}^{2}(\alpha)-\frac{1}{2} I S_{\mathrm{Co}}^{2}(\mathrm{P} \downarrow \downarrow)$ from the calculated DFT dispersion, we arrive at the energy dispersion [squares in Fig. 3(a)], which reflects only exchange interactions between the atoms. If we fit this curve according to Eq. (18) [dashed line in Fig. 3(a)], we obtain the "nonrenormalized" Heisenberg exchange constants of $J_{\mathrm{Co}}=-170 \mathrm{meV}$ and $J_{\mathrm{Mn}}=-366 \mathrm{meV}$. It becomes clear now that, although the "pure" exchange coupling between the Mn and Co spins is expectedly antiferromagnetic, the larger spin moment of Co in the parallel spin alignment with Mn tips the balance in favor of ferromagnetic coupling between the spins, which can be observed for a large range of distances $d_{\mathrm{Mn}-\mathrm{Mn}}$ [cf. Fig. 2(a)].

In Fig. 2(a), we also observe that, judging from the energies, in the close contact regime the collinear $\mathrm{P} \downarrow \uparrow$ state is competing with the noncollinear $\mathrm{P} \alpha$ state for the global ground state of the system. Indeed, our calculations show that at the $d_{\mathrm{Mn}-\mathrm{Mn}}$ of $4.5 \mathrm{bohr}$, the $\mathrm{P} \downarrow \uparrow$ configuration is by a tiny value of $5 \mathrm{meV}$ lower in energy than the $\mathrm{P} 105^{\circ}$ solution. We argue, however, that the $\mathrm{P} \downarrow \uparrow$ state is not very likely to appear in experiments, given that the Co electrodes are identical. In this case, the adiabatic rise of the intrinsically asymmetric $\mathrm{P} \downarrow \uparrow$ configuration via symmetric noncollinear states can not happen, as the electrodes, initially being in the $\mathrm{P} \uparrow \uparrow$ state when very far from each other, are brought together (see also discussion at the beginning of Sec. III E). Nevertheless, it seems plausible that such state, if observed in experiment, is created via a rapid flip of one of the $\mathrm{Mn}$ atoms in the contact regime during, e.g., a reformation of the lead geometry, or an inelastic current-induced spin-flip process. Our calculations, shown with a dotted line in Fig. 3(a), based on the Heisenberg model extended by the Stoner term of intra-atomic exchange of the Co moments, indicate that once the system enters the $\mathrm{P} \downarrow \uparrow$ state, it is effectively "trapped" there since the $\downarrow$-Mn is energetically quite stable versus deviations in the angle its spin makes with the rest of the spins in the system. Thus, we do not consider any noncollinear states associated with the $\mathrm{P} \downarrow \uparrow$ state in the following.

\section{Ballistic conductance of noncollinear magnetic states of the junction}

In this section, we perform a detailed analysis of the ballistic conductance $G\left(E_{F}\right)$ of the $\mathrm{P} \alpha$ state at fixed distance between $\mathrm{Mn}$ atoms of $4.5 \mathrm{bohr}$. At this distance, we calculate $G\left(E_{F}\right)$ as a function of angle $\alpha$ and present the results in Fig. 3(d). In this plot, we observe that the conductance exhibits a very nontrivial dependence on $\alpha$, originating mainly from the $\Delta_{3}$ orbitals $\left(d_{x z, y z}\right)$, while the $\Delta_{1}$ contribution $\left(s-d_{z^{2}}\right)$ to the conductance $G_{\Delta_{1}}$ remains almost perfectly constant. Surprisingly, the $\Delta_{3}$ conductance almost vanishes for $\alpha$ of about $70^{\circ}$, away from any high-symmetry spin configuration in the junction, suggesting that the dependence of the details of hybridization and electronic structure on the angle between the Mn spins can be rather delicate. In order to analyze this dependence in more detail, as a function of $\alpha$, we plot the energy-dependent conductance $G(E)$ versus the local densities of states (LDOS) of $\mathrm{Mn}$ and NN Co atoms resolved into spin-up and -down contributions with respect to the global spin quantization $z$ axis (Fig. 4). Mainly we focus on the $\Delta_{3}$ contribution to the conductance and the LDOS, and only in the upper $(\mathrm{P} \uparrow \uparrow)$ and lower $(\mathrm{P} \downarrow \downarrow)$ panels of Fig. 4 we show also the total LDOS of the atoms.

The conductance at a given energy $E$ depends on the presence of available states in the LDOS of the atoms at $E$, and on the coupling between these states across the junction, both of which depend on the orientation of the spins with respect to each other. By looking at the LDOS of the atoms presented in Fig. 4 for $\alpha=0^{\circ}$, we can explain the absence of the $\Delta_{4}$ $\left(d_{x y, x^{2}-y^{2}}\right)$ contribution to the conductance: the localized $\Delta_{4}$ states of the Co atoms, which can be seen as pronounced peaks in the LDOS marked with the dashed line in Fig. 4, 


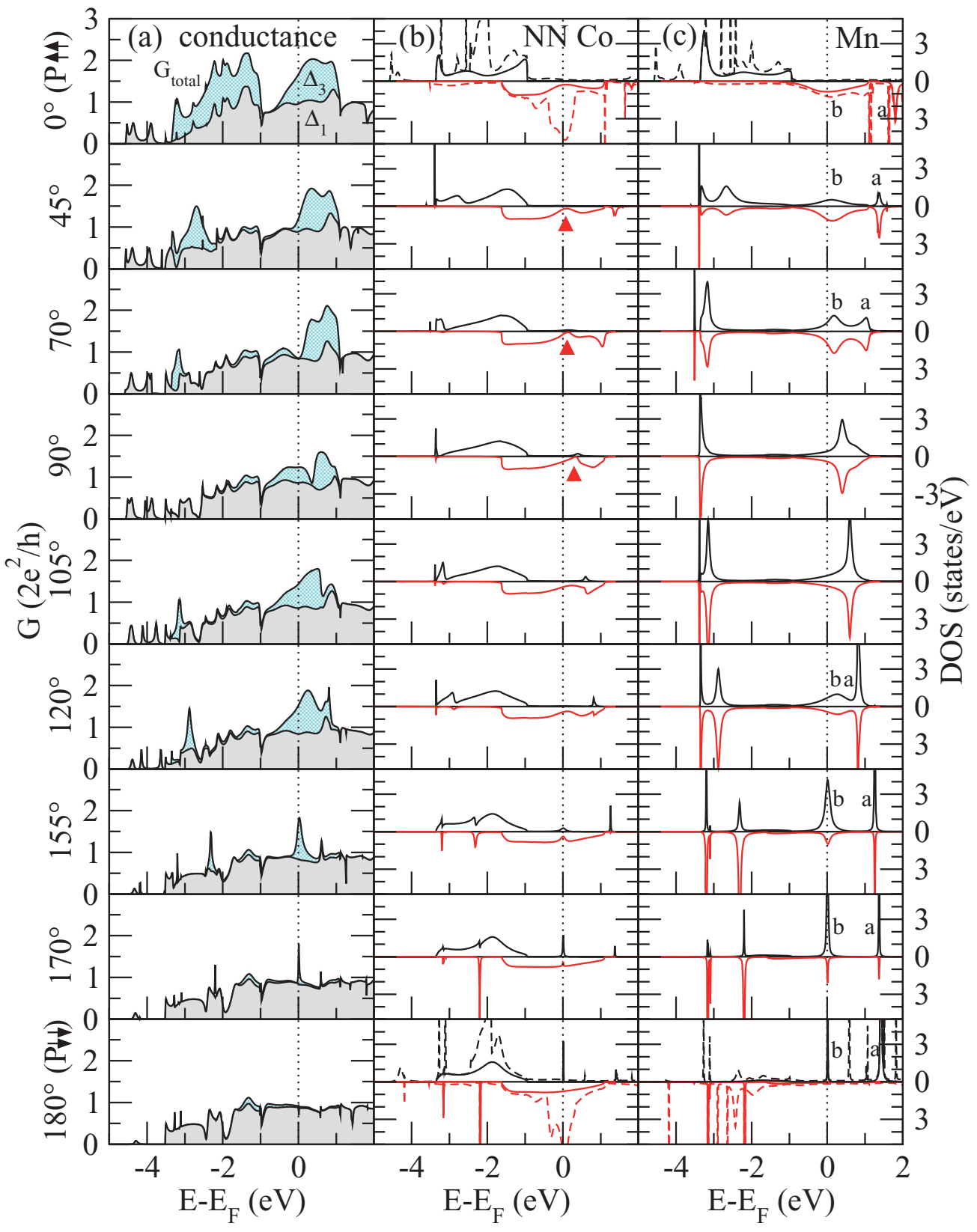

FIG. 4. (Color online) Transport properties and electronic structure of the $\mathrm{P} \alpha$ state at $d_{\mathrm{Mn}-\mathrm{Mn}}$ of 4.5 bohr as a function of angle $\alpha$ (indicated on the left). (a) Total conductance $G_{\text {total }}$ (upper line) decomposed into the $\Delta_{3}$ (cyan shaded area) and $\Delta_{1}$ contributions ( $G_{\Delta_{1}}$, lower line, gray shaded area). The spin-resolved local density of states (LDOS) of the NN Co and Mn atoms are given in columns (b) and (c), respectively. For both (b) and (c), the LDOS spin decomposition is performed with respect to the global frame, with spin-up and -down LDOS presented in the upper and lower parts of each plot. In both (b) and (c), the $\Delta_{3}$ contribution is indicated with solid lines, while for $\alpha=0^{\circ}$ and $180^{\circ}$, also the total LDOS is shown with dashed lines. The red triangles in (b) follow the development of the dip in the LDOS of the NN Co atom as the angle $\alpha$ is varied. In (c), the bonding and antibonding unoccupied Mn states are marked with "b" and "a," respectively. For details, see text.

are positioned at about $-2 \mathrm{eV}$ for the spin-up channel and directly at the Fermi energy for the spin-down channel, while the corresponding $\mathrm{Mn} \Delta_{4}$ states are positioned below -2.5 and above $+1 \mathrm{eV}$, prohibiting thus the hybridization between the Co and Mn orbitals of $\Delta_{4}$ symmetry across the junction. Noticeably, the LDOS of both atoms for the up spin in a wide region of energies around $E_{F}$ is absent, leading to a negligible $\uparrow$ conductance. Here, it is important to remark, that the LDOS of the NN Co atoms around the Fermi energy overall resembles quite well the LDOS of a Co atom in a Co monowire [see, e.g., Fig. 13(a) in Ref. 16] or even of a Co atom deposited on noble-metal surfaces (see, e.g., Ref. 48). This means that our results should be rather stable with respect to the geometry of the Co leads, manifesting that the main influence on the $\Delta_{3}$ conductance at $E_{F}$ would come from the hybridization of the Mn and NN Co states. 
Turning now to the comparatively delocalized $\Delta_{3}$ states (solid line) on both Co and $\mathrm{Mn}$ atoms, we observe for $\mathrm{P} \uparrow \uparrow$ (upper panels of Fig. 4) that they hybridize directly at the Fermi energy, which leads to a significant $\Delta_{3}$ contribution to the conductance. Specifically, while the $\Delta_{3}^{\downarrow}$ subband of Co spreads from -1.8 to $+1 \mathrm{eV}$, the $\Delta_{3}$-down states of $\mathrm{Mn}$ atoms are distinctly split into wide bonding ("b") states at the Fermi energy and narrow antibonding ("a") states at $+1.8 \mathrm{eV}$. Very importantly for the transport properties of the system, the hybridization of the $\Delta_{3}^{\downarrow}$ band of Co with the $\Delta_{3}^{\downarrow}$ states of $\mathrm{Mn}$ is nontrivial. (i) The $\Delta_{3}^{\downarrow}$ states of Co exhibit a dip at the position of the maximal density of bonding states of Mn due to the fact that these Mn states are localized mainly in-between the Mn atoms, prohibiting strong overlap with the Co states. (ii) The upper, antibonding part of the Co $\Delta_{3}^{\downarrow}$ band hybridizes stronger with the bonding states of Mn since the antibonding states of Co atoms have a larger overlap with the Mn orbitals, which results in a larger $\Delta_{3}$ conductance above $E_{F}$. (iii) Analogously, for energies below $E_{F}$ the conductance is suppressed since the bondinglike $\Delta_{3}^{\downarrow}$ Co states have smaller overlap with the Mn bonding states.

Let us now follow the evolution of the electronic structure upon increasing the angle between the Mn spins. Two trends in the LDOS can be clearly observed in Fig. 4. First, with increasing $\alpha$, the splitting between the bonding and antibonding $\mathrm{Mn}$ states decreases owing to the mixed spin character of the states. At the angle of $90^{\circ}$, when Mn spins are antiparallel to each other, both types of states transform into degenerate $\Delta_{3}$ orbitals of the "isolated" Mn atoms since the hybridization between the Mn states of the same spin is almost absent due to large exchange splitting. On the other hand, the dip in the $\downarrow$ LDOS of the NN Co atoms follows the position of the bonding state of the Mn dimer, moving from the Fermi energy at $\alpha=0^{\circ}$ to $+0.2 \mathrm{eV}$ for $\alpha=90^{\circ}$ (indicated by filled triangles in Fig. 4). Overall, such redistribution of the LDOS of the atoms, combined with the effect of decreasing LDOS of Mn atoms for spin-down channel at the Fermi energy when the angle $\alpha$ is varied, results first in a decrease of the conductance at $E_{F}$ for $\alpha \approx 70^{\circ}$, followed by a consequent increase with increasing angle.

When the angle $\alpha$ increases further beyond $90^{\circ}$, the bonding and antibonding $\mathrm{Mn}$ states eventually acquire their initial splitting at $\alpha=180^{\circ}(\mathrm{P} \downarrow \downarrow$ state), when the Mn spins are collinear again. Simultaneously, with increasing angle, we observe that the Mn states around the Fermi energy become sharper since the hybridization with the Co leads decreases as the $\mathrm{Mn}$ states become predominantly spin up in character. Interestingly, while for $90^{\circ}<\alpha<120^{\circ}$ a large value of the $\Delta_{3}$ conductance is due to a significant amount of delocalized Co and bonding $\mathrm{Mn}$ states at the Fermi energy in the spin-down channel, for larger angles the value of $G_{\Delta_{3}}\left(E_{F}\right)$ is due to a sharp resonant Co state in the spin-up channel at the Fermi energy, coupled to a bonding Mn state at $E_{F}$. When further increasing $\alpha$ above $170^{\circ}$, this resonance becomes more localized and decoupled from the states in the leads, while the Mn LDOS at the $E_{F}$ in the minority spin channel vanishes, causing a sharp drop in the $\Delta_{3}$ conductance. By looking at the total LDOS of the NN Co atom in the $\mathrm{P} \downarrow \downarrow$ state, we observe that it remains basically unaffected, as compared to the $\mathrm{P} \uparrow \uparrow$ configuration, while the Mn states become pronouncedly decoupled from the states of the NN Co owing to the energetical mismatch for both spin channels.

\section{E. Fingerprints of noncollinear magnetic states of the junction in ballistic conductance experiments}

Finally, we investigate the evolution of the conductance and the magnetoresistance of different magnetic states of the junction within the contact regime mimicking a typical STM or break-junction experiment. Here, we are partly motivated by the fact that a nontrivial behavior of magnetoresistance when going from tunneling to contact has been recently observed in STM experiments (see, e.g., Ref. 8). At a very large separation between the leads (or, the tip and the sample in the STM language), owing to the FM coupling of the Mn atom to the Co chain, one can imagine only two possible magnetic configurations: $\mathrm{P} \uparrow \uparrow$ and $\mathrm{AP} \uparrow \downarrow$. The conductance of these two magnetic states in the tunneling regime, arising mainly from the $s$ orbitals, is orders of magnitude smaller than in the contact regime, for which the dependence of $G\left(E_{F}\right)$ on the distance can be nontrivial due to the large contribution of the $d$ states.

In the case of the AP $\uparrow \downarrow$ configuration, the starting collinear arrangement of the spins will survive over the whole range of the separation between the leads since, in the contact regime, when the $\mathrm{Mn}$ atoms are close to each other, both exchange preferences of the Mn spins, that is, FM coupling to the NN Co spins and AFM coupling among each other, are fulfilled. Small possible deviations from the collinear arrangement of the Mn spins, which can affect the details of the distribution of the $\Delta_{3}$ states and their coupling to the leads, would not manifest in a conductance measurement, owing to the antiparallel magnetizations of the leads, and corresponding complete dominance of the $\Delta_{1}$ channel for conductance at $E_{F}$ in this case (Fig. 5). As we can see from this figure, $G\left(E_{F}\right)$ lies in-between 0.5 and $1.0 G_{0}$, when the distance between the $\mathrm{Mn}$ atoms is varied from 8.5 to $4.5 \mathrm{bohr}$. This is very similar to the behavior of the conductance at the Fermi energy of pure AP Co leads without Mn atoms [see, e.g., Fig. 11(b) of Ref. 16].

Owing to the magnetic frustration of the Mn spins of the junction in the contact regime, for the $\mathrm{P} \uparrow \uparrow$ initial configuration, we consider the $\mathrm{P} \alpha$ and $\mathrm{P} \downarrow \uparrow$ states in addition to the $\mathrm{P} \uparrow \uparrow$ state when the Mn-Mn distance is relatively small. Here, as we have seen in the preceding section, the conductance at the Fermi energy can be very strongly influenced by the details of hybridization between the $\Delta_{3}$ orbitals. On the other hand, since very often transport measurements serve as the only experimental insight into the magnetic structure of a system, it is very important to coin each of the possible magnetic states with a unique fingerprint which can be related to the experimental data. In the following, we suggest that indeed three distinct spin states in a $\mathrm{Co}_{\infty}-\mathrm{Mn}-\mathrm{Mn}-\mathrm{Co}_{\infty}$ junction, which can occur in an experiment due to various reasons such as structural details, temperature fluctuations, external magnetic field, etc., lead to different transport signatures.

As already shown in Fig. 2(d), the conductance of the collinear $\mathrm{P} \uparrow \uparrow$ state rapidly rises towards a value of $1.8 G_{0}$ as the distance between the leads is decreased. Compared to other possible magnetic configurations of the junction, 


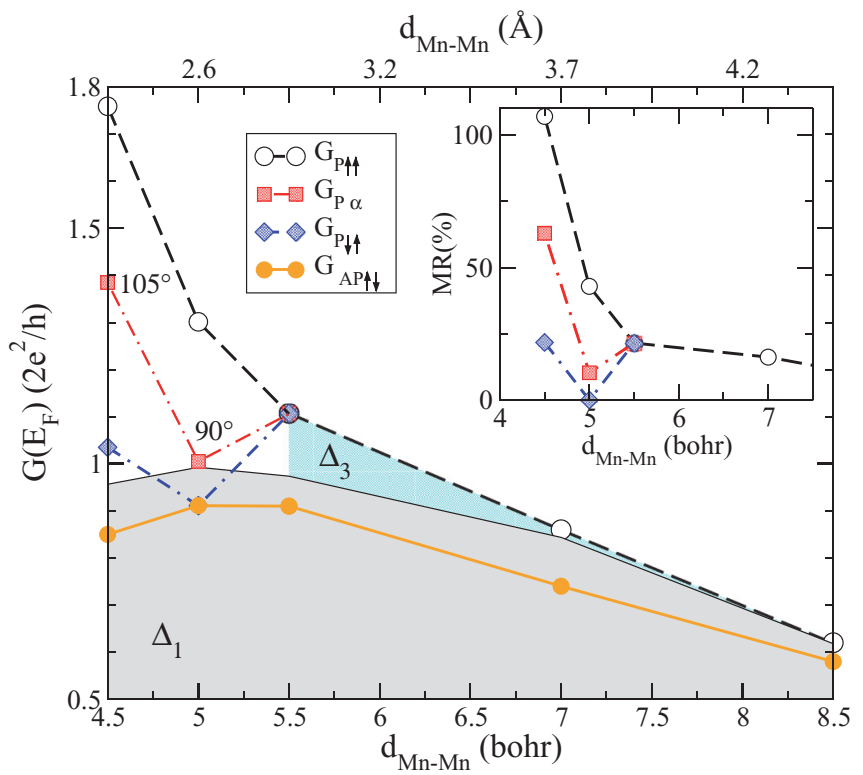

FIG. 5. (Color online) Conductance at the Fermi energy of various possible magnetic states of the $\mathrm{Co}_{\infty}-\mathrm{Mn}-\mathrm{Mn}-\mathrm{Co}_{\infty}$ junction as a function of the distance between the Mn atoms. The following magnetic states are considered: $\mathrm{AP} \downarrow \uparrow$ (filled circles, solid line), $\mathrm{P} \uparrow \uparrow$ (open circles, dashed line), $\mathrm{P} \downarrow \uparrow$ (diamonds, dashed-dashed-dotted line), and $\mathrm{P} \alpha$ (squares, dotted-dashed line). The $\Delta_{1}$ contribution for the $\mathrm{P} \uparrow \uparrow$ state is shown with a thin solid line and gray shaded area, while the $\Delta_{3}$ part is shaded in cyan. For the $\mathrm{P} \alpha$ states, the state which is lowest in energy among all possible angles $\alpha$ at a fixed distance is considered. In the inset, the values of the magnetoresistance for different $\mathrm{P}$ states are shown.

$G_{\mathrm{P} \uparrow \uparrow}\left(E_{F}\right)$ is significantly larger in value (see Fig. 5) because of the alignment of the minority spin $s$ and $d$ states of the Co electrodes and the Mn atoms at the Fermi energy, which ideally favors perfect transmission. In contrast, the conductance of the collinear $\mathrm{P} \downarrow \uparrow$ state is significantly suppressed, reaching only $1.0 G_{0}$ at the separation of $4.5 \mathrm{bohr}$, due to the large exchange splitting of the $\Delta_{3}$ states of the Mn atoms with antiparallel spin moments, which hinders the $\Delta_{3}$ conductance. A small dip in the conductance at $5.0 \mathrm{bohr}$ for this configuration is due to the promotion of the second-nearest-neighbor ferromagnetic CoMn hopping across the antiparallel Mn spin with decreasing distance between the Mn atoms.

The conductance of the noncollinear ground-state $\mathrm{P} \alpha$ state lies in-between the values for both limiting collinear configurations. In the close contact regime, at $d_{\mathrm{Mn}-\mathrm{Mn}}$ of $4.5 \mathrm{bohr}$, the conductance of the $\mathrm{P} \alpha$ state of $1.4 G_{0}$ is exactly in-between the values of $G_{\mathrm{P} \uparrow \uparrow}\left(E_{F}\right)$ and $G_{\mathrm{P} \downarrow \uparrow}\left(E_{F}\right)$. Clearly, the difference of $0.4 G_{0}$, stemming from the variation in the $\Delta_{3}$ conductance with the spin state, can be easily detected in experiment, allowing for a way to distinguish between different possible magnetic configurations. At the distance of $5.0 \mathrm{bohr}$, the ground state among the $\mathrm{P} \alpha$ states is the $\mathrm{P} 90^{\circ}$ state, while at larger distances above $5.5 \mathrm{bohr}$, the system converges to a collinear configuration. The angle $\alpha$ in the lowest in energy $\mathrm{P} \alpha$ state decreases smoothly with increasing the separation, and we speculate, that owing to the nonmonotonous behavior of the conductance as a function of $\alpha$, seen in Fig. 3(d), the conductance as a function of $d_{\mathrm{Mn}-\mathrm{Mn}}$ can exhibit several dip features similar to that at $d_{\mathrm{Mn}-\mathrm{Mn}}$ of $5.0 \mathrm{bohr}$, although we did not perform the calculations to support this statement owing to the required computational effort.

According to recent experiments, ${ }^{8}$ the conductance of the junction with the parallel (P) and antiparallel (AP) orientations of the lead's magnetization can be related to each other via measuring the magnetoresistance (MR). From the values presented in Fig. 5, we calculate the MR of the junction, defined as

$$
\mathrm{MR}=\frac{G_{\mathrm{P}}\left(E_{F}\right)-G_{\mathrm{AP}}\left(E_{F}\right)}{G_{\mathrm{AP}}\left(E_{F}\right)} \times 100 \%,
$$

and present the MR as a function of separation between the electrodes in the inset of Fig. 5, where we choose $G_{\mathrm{AP} \downarrow \uparrow}$ for $G_{\mathrm{AP}}\left(E_{F}\right)$, and values of $G_{\mathrm{P} \uparrow \uparrow}\left(E_{F}\right), G_{\mathrm{P} \downarrow \uparrow}\left(E_{F}\right)$, and $G_{\mathrm{P} \alpha}\left(E_{F}\right)$ for $G_{\mathrm{P}}\left(E_{F}\right)$. The overall smaller $\mathrm{AP} \downarrow \uparrow$ conductance as compared to the $\mathrm{P}$ configurations results in positive magnetoresistance values. The MR curves as a function of the distance generally resemble those of the conductance, with the values of the MR of 22,62, and $105 \%$ at the distance of 4.5 bohr for the $\mathrm{P} \downarrow \uparrow, \mathrm{P} \alpha$, and $\mathrm{P} \uparrow \uparrow$ states, respectively. Much more pronounced in the MR is the feature characteristic to the $\mathrm{P} \downarrow \uparrow$ and $\mathrm{P} \alpha$ configurations, a dip around the distance of $5.0 \mathrm{bohr}$, also present in the conductance curves. As can be seen from Fig. 5, at this distance, the MR almost completely vanishes when the Mn spins exhibit a different from FM configuration. Overall, we conclude that the pronounced difference in the shape and magnitude of the MR curves can be also used in experiments such as those performed in Ref. 8 to shed light onto the complex magnetism in this type of systems.

\section{SUMMARY}

In this work, we presented the realization of a firstprinciples scheme for calculating the ballistic transport properties of magnetically complex one-dimensional systems employing the technique of noncollinear Wannier functions. We use the FLAPW method in order to calculate the electronic structure of the system with high accuracy and use the Wannier functions to transfer it to our transport calculations performed within the Landauer approach. As spin-orbit interaction can be naturally included into the consideration within this technique (cf. Ref. 16), the method introduced here can be used to explore the rich field of transport phenomena in systems such as nanosized or atomic-sized contacts, break junctions, or STM experiments for which both effects, spin-orbit coupling and frustrated exchange interactions, can be prominent.

As a first application of our approach, we consider the ballistic transport properties of a single-atom junction formed by two semi-infinite Co electrodes with a single-apex $\mathrm{Mn}$ atom. We study the conductance as a function of the separation between the two $\mathrm{Mn}$ atoms from the tunneling to the contact regime, taking into account the complex magnetic interaction in the junction. As we demonstrate, even such a simple setup allows us to draw some general conclusions concerning the interplay of structure and magnetism for the transport through such atomic-sized contacts, which are in the focus of 
today's research. We analyze the ballistic conductance of the junction with lead magnetizations in parallel and antiparallel alignments. We consider separately the tunneling (separation larger than about $5 \AA$ ) and contact (below $5 \AA$ ) regimes of the junction, and we demonstrate that in the tunneling regime the conductance $G$ is solely coming from the overlap between the $\Delta_{1}\left(s-d_{z^{2}}\right.$ orbitals) of the contacts. In this case, the Mn spins prefer to order ferromagnetically with respect to the magnetization of the leads. On the other hand, upon reaching the close contact regime (below $3.5 \AA$ ), when the magnitude of the conductance reaches $1 G_{0}$, the hybridization between the $\Delta_{3}\left(d_{x z}, d_{y z}\right.$ orbitals $)$ states of the junction starts to provide a sizable contribution to $G$.

In the close contact regime, when the hybridization between the $\mathrm{Mn}$ atoms is significant, Mn spins experience a frustration due to the FM coupling with the leads and an AFM Mn-Mn coupling. The competition between the two gives rise to a stable noncollinear solution which can be characterized by a tilting angle of the spins $\alpha$. General for this type of junction is the sensitivity of the $d$-orbital conductance on the angle $\alpha$, which is due to a delicate interplay between the hybridization details of the Mn and Co states at the Fermi energy, as well as spin asymmetry in their distribution. This gives rise to a nontrivial $\alpha$ dependence of the conductance of the $d$ states. We show that the complicated $\Delta_{3}$-channel conductance arising on the background of almost constant $\Delta_{1}$ contribution can be used in order to distinguish between different magnetic states of the contact via either a direct conductance measurement or via measuring the magnetoresistance, which, according to our calculations, can vary in the contact regime between $20 \%$ and $100 \%$, depending on the spin arrangement.

Finally, we would like to comment on our approximation for the geometry of the junction we have assumed in this work. Albeit being very simple, it allows us to capture the key features which govern the transport properties of the system, while keeping the computational burden reasonable. Namely, within this geometry (i) the transition from tunneling to contact can be naturally studied, (ii) the magnetic frustration of the spins in the junction and (iii) the delicate details of the hybridization of the adatom with the lead reservoirs are taken into account, and (iv) the sensitive dependence of the spin moments on the magnetic configuration in the nanocontact is included into our considerations. Of course, in order to achieve a quantitative agreement of the calculated values to the experimentally measured ones in this type of junction beyond the major trends, all details of the structure and structural reformation upon approaching should be ideally accounted for. Such a challenging study lies, however, outside of the scope of this work, and we leave it for future studies.

\section{ACKNOWLEDGMENTS}

We acknowledge helpful discussions with S. Blügel. Funding by the DFG within the SFB677 is gratefully acknowledged. S.H. thanks the DFG for financial support under HE3292/8-1. Y.M. and F.F. gratefully acknowledge the Jülich Supercomputing Centre for computing time and funding under the HGF-YIG Programme VH-NG-513.
*Corresponding author: y.mokrousov@fz-juelich.de

${ }^{1}$ A. I. Yanson, G. R. Bollinger, H. E. van den Brom, N. Agrait, and

J. M. van Ruitenbeek, Nature (London) 395, 783 (1998).

${ }^{2}$ A. Thiess, Y. Mokrousov, S. Blügel, and S. Heinze, Nano Lett. 8, 2144 (2008).

${ }^{3}$ N. Néel, J. Kröger, and R. Berndt, Phys. Rev. Lett. 102, 086805 (2009).

${ }^{4}$ J. Kröger, N. Néel, and L. Limot, J. Phys.: Condens. Matter 20, 223001 (2008).

${ }^{5}$ K. Tao, I. Rungger, S. Sanvito, and V. S. Stepanyuk, Phys. Rev. B 82, 085412 (2010).

${ }^{6}$ H. D. Chopra, M. R. Sullivan, J. N. Armstrong, and S. Z. Hua, Nat. Mater. 4, 832 (2005).

${ }^{7}$ M. Calvo, J. Fernández-Rossier, J. Palacios, D. Jacob, D. Natelson, and C. Untiedt, Nature (London) 458, 1150 (2009).

${ }^{8}$ M. Ziegler, N. Néel, C. Lazo, P. Ferriani, S. Heinze, J. Kröger, and R. Berndt, New J. Phys. 17, 085011 (2011).

${ }^{9}$ S. Schmaus, A. Bagrets, Y. Nahas, T. K. Yamada, A. Bork, M. Bowen, E. Beaurepaire, F. Evers, and W. Wulfhekel, Nat. Nanotechnol. 6, 185 (2011).

${ }^{10}$ M. R. Calvo, J. Fernández-Rossier, J. J. Palacios, D. Jacob, D. Natelson, and C. Untiedt, Nature (London) 458, 1150 (2009).

${ }^{11}$ A. Delin and E. Tosatti, Phys. Rev. B 68, 144434 (2003).

${ }^{12}$ A. Smogunov, A. Dal Corso, A. Delin, R. Weht, and E. Tosatti, Nat. Nanotechnol. 3, 22 (2008).
${ }^{13}$ A. Delin, E. Tosatti, and R. Weht, Phys. Rev. Lett. 92, 057201 (2004).

${ }^{14}$ A. Thiess, Y. Mokrousov, S. Heinze, and S. Blügel, Phys. Rev. Lett. 103, 217201 (2009).

${ }^{15}$ A. Thiess, Y. Mokrousov, and S. Heinze, Phys. Rev. B 81, 054433 (2010).

${ }^{16}$ B. Hardrat, N.-P. Wang, F. Freimuth, Y. Mokrousov, and S. Heinze, Phys. Rev. B 85, 245412 (2012).

${ }^{17}$ A. Smogunov, A. Dal Corso, and E. Tosatti, Phys. Rev. B 70, 045417 (2004).

${ }^{18}$ A. Smogunov, A. Dal Corso, and E. Tosatti, Phys. Rev. B 73, 075418 (2006).

${ }^{19}$ A. Bagrets, N. Papanikolaou, and I. Mertig, Phys. Rev. B 70, 064410 (2004).

${ }^{20}$ A. Bagrets, N. Papanikolaou, and I. Mertig, Phys. Rev. B 75, 235448 (2007).

${ }^{21}$ M. Polok, D. V. Fedorov, A. Bagrets, P. Zahn, and I. Mertig, Phys. Rev. B 83, 245426 (2011).

${ }^{22}$ J. D. Burton, R. F. Sabirianov, S. S. Jaswal, E. Y. Tsymbal, and O. N. Mryasov, Phys. Rev. Lett. 97, 077204 (2006).

${ }^{23}$ M. Czerner, B. Y. Yavorsky, and I. Mertig, Phys. Rev. B 77, 104411 (2008).

${ }^{24}$ M. Czerner, B. Y. Yavorsky, and I. Mertig, Phys. Status Solidi B 247, 2594 (2010).

${ }^{25}$ A. Smogunov, A. Dal Corso, and E. Tosatti, Phys. Rev. B 78, 014423 (2008). 
${ }^{26}$ J. Velev, R. F. Sabirianov, S. S. Jaswal, and E. Y. Tsymbal, Phys. Rev. Lett. 94, 127203 (2005).

${ }^{27}$ K. I. Bolotin, F. Kuemmeth, and D. C. Ralph, Phys. Rev. Lett. 97, 127202 (2006).

${ }^{28}$ J. D. Burton, R. F. Sabirianov, J. P. Velev, O. N. Mryasov, and E. Y. Tsymbal, Phys. Rev. B 76, 144430 (2007).

${ }^{29}$ K. Tao, V. S. Stepanyuk, W. Hergert, I. Rungger, S. Sanvito, and P. Bruno, Phys. Rev. Lett. 103, 057202 (2009).

${ }^{30}$ D. Serrate, P. Ferriani, Y. Yoshida, S.-W. Hla, M. Menzel, K. von Bergmann, S. Heinze, A. Kubetzka, and R. Wiesendanger, Nat. Nanotechnol. 5, 350 (2010).

${ }^{31}$ M. Menzel, Y. Mokrousov, R. Wieser, J. E. Bickel, E. Vedmedenko, S. Blügel, S. Heinze, K. von Bergmann, A. Kubetzka, and R. Wiesendanger, Phys. Rev. Lett. 108, 197204 (2012).

${ }^{32}$ P. Hohenberg and W. Kohn, Phys. Rev. 136, B864 (1964).

${ }^{33}$ P. Kurz, F. Förster, L. Nordström, G. Bihlmayer, and S. Blügel, Phys. Rev. B 69, 024415 (2004).

${ }^{34}$ G. H. Wannier, Phys. Rev. 52, 191 (1937).

${ }^{35}$ N. Marzari and D. Vanderbilt, Phys. Rev. B 56, 12847 (1997).
${ }^{36}$ F. Freimuth, Y. Mokrousov, D. Wortmann, S. Heinze, and S. Blügel, Phys. Rev. B 78, 035120 (2008).

${ }^{37}$ www.flapw.de

${ }^{38}$ H. Krakauer, M. Posternak, and A. J. Freeman, Phys. Rev. B 19, 1706 (1979).

${ }^{39}$ Y. Mokrousov, G. Bihlmayer, and S. Blügel, Phys. Rev. B 72, 045402 (2005)

${ }^{40}$ T. Kizuka, Phys. Rev. B 77, 155401 (2008).

${ }^{41}$ Y. Zhang and W. Yang, Phys. Rev. Lett. 80, 890 (1998).

${ }^{42}$ U. Birkenheuer and D. Izotov, Phys. Rev. B 71, 125116 (2005).

${ }^{43}$ Y. Mokrousov, G. Bihlmayer, S. Blügel, and S. Heinze, Phys. Rev. B 75, 104413 (2007).

${ }^{44}$ F. Schubert, Y. Mokrousov, P. Ferriani, and S. Heinze, Phys. Rev. B 83, 165442 (2011).

${ }^{45}$ M. Zelený, M. Šob, and J. Hafner, Phys. Rev. B 80, 144414 (2009).

${ }^{46}$ C. Ataca, S. Cahangirov, E. Durgun, Y.-R. Jang, and S. Ciraci, Phys. Rev. B 77, 214413 (2008).

${ }^{47}$ O. Gunnarsson, J. Phys. F: Met. Phys. 6, 587 (1976).

${ }^{48}$ B. Nonas, I. Cabria, R. Zeller, P. H. Dederichs, T. Huhne, and H. Ebert, Phys. Rev. Lett. 86, 2146 (2001). 